\title{
Comparison of a modified microagglutination technique for HBsAg with the standard technique and a new RIA system
}

\author{
E MARGARET SUPRAN, PS GARDNER, I CAYZER, * G COURT,* \\ S CHALMERS* \\ From the Division of Microbiological Reagents and Quality Control, Central Public Health \\ Laboratory, 175 Colindale Avenue, London NW9 5HT and *Wellcome Research Laboratories, \\ Beckenham, Kent BR3 $3 B S$
}

SUMMARY A further modification of the standard RPHA technique (Hepatest, Wellcome Reagents) for the detection of $\mathrm{HBsAg}$ is described. This modification does not require a centrifugation step which is required by the other modifications that have been described previously and consequently takes a little longer to perform. It does, however, retain the advantages of increased sensitivity and decreased costs which are also features of the other modifications. A series of 939 routine clinical specimens were used to evaluate the method described and to evaluate a new RIA kit for the detection of HBsAg (Hepatube, Wellcome Reagents). Of 53 specimens found to be positive for $\mathrm{HBsAg}$ by RIA, $50(94 \%)$ were detected by the modified Hepatest RPHA as opposed to $47(89 \%)$ by the standard technique.

A modification of the Hepatest RPHA technique (Wellcome Reagents) has been described ${ }^{1}$ suitable for the rapid large scale screening of blood donor samples for HBsAg. This modification resulted in a slight increase in sensitivity and a saving in the cost of reagents. The technique involved the dilution of the Hepatest cells to $0.2 \%$, the use of V-bottomed microtitre plates rather that U-bottomed plates recommended by the manufacturer and the inclusion of extra turkey serum in the buffer to diminish the number of false positive reactions which would be given by those sera with low level turkey agglutinins if the test were made more sensitive. An important and necessary step was the centrifugation of the plates $(15 \mathrm{~s}$ at $450 \mathrm{~g})$ after incubation with the test sera to hasten the agglutination of the cells. After centrifugation the plates were inclined at an angle of $60^{\circ}$ for a few minutes when the negative unagglutinated cells ran down as a smooth teardrop streak. A further modification to this technique using $0.1 \%$ test cells, was later described ${ }^{2}$ with a similar increase in sensitivity over the standard technique, but because of the centrifugation step the test could still be performed within $30 \mathrm{~min}$.

Many laboratories, however, are not required to

Accepted for publication 27 May 1981 process the large number of specimens per day that is usual in a large blood bank and therefore the time taken to perform the test is not so critical. In addition, a general diagnostic laboratory may not have special facilities for centrifuging plates containing hepatitis B-positive specimens, HBV still being classified as a Category B pathogen. We here report a small trial employing a similar modified Hepatest technique which omits the centrifugation step, and although it takes a little longer to perform, still retains the advantages of increased sensitivity and lower cost.

The sera tested were also used to evaluate the new RIA kit for the detection of HBsAg (Hepatube) supplied by Wellcome Reagents.

\section{Material and methods}

Nine hundred and thirty nine sera were received from the Portsmouth Public Health Laboratory. These represented routine specimens for diagnosis over a two-month period and included a batch of 92 specimens from Vietnamese refugees (Boat People). The sera (under code) were screened for the presence of HBsAg by Hepatest RPHA according to the manufacturer's instructions (the standard method) and by the modification described below (the modified method). The same batch of Hepatest was used throughout. In addition all specimens were 
tested by radioimmunoassay for HBsAg (Hepatube, Wellcome Reagents).

HEPATEST RPHA-STANDARD METHOD

In the presence of $\mathrm{HBs} A g$, horse anti-HBs-coated turkey erythrocytes are agglutinated. ${ }^{3}$ Freeze-dried antibody-coated cells and control cells coated with normal horse immunoglobulin are supplied which when reconstituted form a $1 \%$ suspension. Buffer and positive and negative control sera are supplied, and the test is performed in U-bottomed microtitre plates using $25 \mu \mathrm{l}$ as the unit volume for serum dilutions and cells. After the test is set up, the plates are left at room temperature for $30 \mathrm{~min}$ before the agglutination patterns are read.

The test is negative if the cells settle into a tight ring or button, and positive if the cells form a smooth carpet over the bottom of the well. In our trial all specimens were screened initially at a dilution of $1 / 8$ with test cells only, any positive specimens then being titrated with test and control cells to ensure that the reaction was specific.

Before a batch of Hepatest is accepted for use by the Public Health Laboratory Service it is subjected to quality control by the Division of Microbiological Reagents and Quality Control at Colindale and by the Leeds Public Health Laboratory. Sensitivity assays include screening tests with the special lowtitred HBsAg panel (the "SP Panel") when at least the seven highest titred antigens must be detected; and titrations of a panel of 10 reagents (including negative sera and true and false positive sera) when minimum titres must be obtained. The settling of the turkey cells must not be unduly prolonged but give clear and distinct positive and negative reactions.

\section{HEPATEST RPHA-MODIFIED METHOD}

The buffer supplied with the Hepatest kit was supplemented with $2 \%$ normal turkey serum. Dilutions of the specimen were made in this buffer which was also used to dilute the $1 \%$ test and control suspensions to $0 \cdot 2 \%$. Screening tests were set up as in the standard method with the same unit volumes, but diluted turkey suspensions and V-bottomed microtitre plates were used. The plates were left undisturbed in a horizontal position for $1 \frac{1}{2}$ hours at room temperature to allow the diluted Hepatest cells to settle before the agglutination patterns were read. The plates may be read from below by means of a viewing mirror if desired. Again, a tight button of cells constituted a negative reaction and a carpet of agglutniated cells a positive reaction. When used to screen the SP Panel, the positives detected by the standard method were seen to be more clearly positive by the modified method and two additional antigens were also detected.
A second modification was also tried and used in parallel with the modification described above on approximately $10 \%$ of the sera. As this alternative modification is a 3-volume test it was necessary only to supplement the buffer supplied in the kit with $1 \%$ normal turkey serum to overcome any increase in non-specific agglutination due to the use of diluted cell suspensions. Dilutions of the specimens were made in this buffer which was also used to dilute the $1 \%$ test and control cells to $0 \cdot 15 \%$. Screening tests at a $1 / 8$ serum dilution were set up as in the standard procedure but flexible PVC V-well microtitre plates were used instead of the rigid U-well polystyrene plates. To each $25 \mu \mathrm{l}$ of serum dilution was added $2 \times 25 \mu l$ of the $0.15 \%$ cell suspension and the plates left at room temperature for $1 \frac{1}{2}$ to 2 hours before the cell patterns were read. Again a tight button of cells constituted a negative, and a carpet of agglutinated cells, a positive reaction.

Both modifications performed equally well giving the same increased sensitivity. There was however a slight improvement in the clarity of the settling patterns using the second modification but as the flexible PVC plates were more expensive, not so robust and more subject to vibrations than the rigid V-bottomed polystyrene plates, this modification although it can be recommended, was not the chosen modification used in this survey.

HEPATUBE-RIA

This "sandwich principle" radioimmunoassay employs antibody-coated plastic tubes as the solid phase, the antibody being guinea-pig anti-HBs. Volumes $(0.2 \mathrm{ml})$ of the sera under test were added to the tubes and any HBsAg present was fixed during the initial incubation. For convenience an overnight incubation at room temperature was used at this stage instead of the two hours at $37^{\circ} \mathrm{C}$ recommended in the assay protocol. After washing, $0.2 \mathrm{ml}{ }^{125}$ I-labelled horse anti-HBs was added and the tubes incubated for one hour at $37^{\circ} \mathrm{C}$, before being washed again and the radioactivity counted. Three positive and six negative replicate controls were included in each test. A positive was defined as any sample giving a count $60 \mathrm{cpm}$ above the mean negative count rate.

\section{Results}

The total number of specimens positive by RPHA was 50 . The number positive by the standard method was 47 , whereas the modified test detected 50 positives. The three specimens not detected by the standard method were all very weak positives with titres of $1 / 8$ (two specimens) and 1/16 (one specimen) 
in the modified test.

There were only two repeatable false positive RPHA reactions out of 939 sera tested, both given by the modified technique. This is an incidence of $0.2 \%$. In this study RIA (Hepatube) was used as the confirmatory test.

Sufficient sera were available for $25 \mathrm{HBsAg-}$ positive patients for titrations by the standard and modified RPHA methods to be carried out in parallel. Six specimens showed an eightfold higher titre by the modified method than by the standard method, 10 specimens showed a fourfold higher titre, eight specimens a twofold higher titre and one specimen gave the same titre by both methods.

All 939 sera were tested on the one occasion for the presence of HBsAg by RIA (Hepatube). There were 53 specimens positive by this method, the 50 specimens positive by the modified RPHA were confirmed and an extra three positives detected. These three patients were British subjects who had had proven hepatitis B a few months previously and who were now negative for HBsAg by RPHA. When tested for anti-HBc by RIA (test kindly performed by Dr BJ Cohen, Virus Reference Laboratory, Colindale), all three were positive. All positives were also tested by Ausria II (Abbott Labs) as a further confirmatory test and found to be positive. Hepatube was shown to be at least as sensitive as Ausria II for the detection of HBsAg as both were equally capable of detecting all 16 positive samples in the low-titre HBsAg panel (SP Panel) held by Division of Microbiological Reagents and Quality Control.

Among the 92 sera from the Vietnamese Boat People, $24(26 \%)$ were positive for $\mathrm{HBsAg}$, therefore there were 29 positives among the remaining 847 hospital specimens; an incidence of $3.4 \%$.

\section{Discussion}

Previous workers, notably in the Blood Transfusion Service $^{12}$ have modified the standard Hepatest technique in the interest of economy and sensitivity but have retained the advantage of the speed of the test by introducing a centrifugation step to speed up the settling of the cells. This is an essential step in any modification involving the dilution of the turkey cells if the rapidity of the test is to be preserved. The justification for yet another modification of the Hepatest technique is that it allows workers in less specialised laboratories with a smaller hepatitis work load to take advantage of the benefits of a modified test-increased sensitivity and decreased costs-even if for reasons of safety or because they lack the necessary apparatus, they are not able to include the centrifugation step. No additional technical complications are introduced in the method described, the modifications being made in the buffer, the dilution of the Hepatest cells, the agglutination plates used and the time of incubation of the plates. Thus this method could be adopted as a routine test in most general laboratories. There are few clinical demands in which the extra incubation period would make any significant difference.

That the sensitivity of Hepatest RPHA is increased by the modification described is shown by the fact that of 53 specimens positive for HBsAg by RIA, $50(94 \%)$ were detected by the modified version as opposed to $47(89 \%)$ by the standard method. It must be emphasised that any suspicious finding on screening should result in that specimen being titrated with both test and control cells, and any unexpected or weak positive reactions should be checked by RIA, if necessary by reference to another laboratory.

Another major benefit is the more economical use of the reagents required to carry out the test. Extra buffer and normal turkey serum will need to be purchased but this is more than offset by the fact that the Hepatest cells are diluted five times before use. Thus the saving in cost could be as much as four to five times.

All specimens were able to be tested in one da by RIA using Hepatube reagents and automate washing equipment provided by Wellcome Reagents. The test performed well and appeared to be as sensitive as Ausria II. Among the 939 hospital specimens, Hepatube detected an extra three positive specimens overall in addition to those detected by RPHA. This was $11 \%$ more than those detected by the standard Hepatest but only $5 \cdot 6 \%$ more than those detected by the modified method. A similar increase in sensitivity by RIA over Hepatest was reported among 25000 new blood donors at the North London Blood Transfusion Centre. ${ }^{2}$ RIA (Middlesex Hospital technique) $^{2}$ detected $11 \%$ more positives than did the standard Hepatest technique, but this was reduced to $6 \%$ when their $0 \cdot 1 \%$ Hepatest was used. This similarity of findings is in two very different populations with very different HBsAg incidences. The positivity rate in our small series of hospital patients and Vietnamese refugees together being 1:18 compared with $1: 485$ among the apparently healthy blood donor population.

We are grateful to Dr AAG Saeed of the Portsmouth Public Health Laboratory for providing us with the specimens tested. 
References

1 Archer AC. An improved haemagglutination technique for the detection of hepatitis B antigen. Med Lab Sci 1977; 34:345-50.

2 Barbara JAJ, Harrison PJ, Howell DR, et al. A sensitive single reverse passive haemagglutination test for detecting both HBsAg and anti-HBs. Vox Sang 1979;32:4-9.

${ }^{3}$ Cayser I, Dane DS, Cameron CH, Denning JV. A rapid haemagglutination test for hepatitis-B antigen. Lancet $1974 ; \mathrm{i}: 947-9$.

Requests for reprints to: Dr PS Gardner, Director, Central Public Health Laboratory, Division of Microbiological Reagents and Quality Control, 175 Colindale Avenue, London NW9 5HT, England.

\section{The November 1981 issue}

THE NOVEMBER 1981 ISSUE CONTAINS THE FOLLOWING PAPERS FROM THE ACP SYMPOSIUM “DISEASES OF THE KIDNEY” HELD IN LONDON, NOVEMBER, 1980.

\section{Editors' foreword}

New ideas on the anatomy of the kidney DB MOFFAT

Advances in understanding the morphology of glomerular disease DR TURNER

Role of circulating immune complexes in renal diseases RJ LEVINSKY

New ideas in the pathogenesis of nephritis D GWYN WILLIAMS

\section{Renal amyloidosis NF JONES}

Blood pressure and the kidney JD SWALES

The kidney and liver diseases SP WILKINSON

Uric acid, gout and the kidney JS CAMERON, HA SIMMONDS
Analgesic nephropathy in the United Kingdom: incidence, clinical features and pathogenesis JR COVE-SMITH

Genesis and evolution of diabetic nephropathy H KEEN, GC VIBERTI

Modern diuretics and the kidney AF LANT

Old and new tests of renal function GB HAYCOCK

New ways in dialysis RA BAILLOD

Dialysis encephalopathy, bone disease and anaemia: the aluminium intoxication syndrome during regular haemodialysis IS PARKINSON, MK WARD, DNS KERR

Osteomalacia and chronic renal failure JA KANIS

\section{Letters to the Editor}

\section{Book reviews}

Copies are still available and may be obtained from the PUBLISHING MANAGER, BRITISH MEDICAL ASSOCIATION, TAVISTOCK SQUARE, LONDON WC1H 9JR, price $£ 3.00$, including postage 